\title{
Diagnostic, Therapeutic, or Research Equipment
}

National Cancer Institute

\section{Source}

National Cancer Institute. Diagnostic, Therapeutic, or Research Equipment. NCI

Thesaurus. Code C19238.

A manufactured object that is used to perform diagnostic, therapeutic or research activities. 